\title{
Near Process Coolant Flow Field Measurements in a Grinding Machine
}

\author{
C. Vanselow ${ }^{1}$, B. Espenhahn ${ }^{1}$, L. Schumski ${ }^{2}$, D. Stöbener ${ }^{1,3}$, D. Meyer ${ }^{2,3}$, A. Fischer ${ }^{1,3}$ \\ 1 University of Bremen, Bremen Institute for Metrology, Automation and \\ Quality Science (BIMAQ), Linzer Str. 13, 28359 Bremen, Germany, \\ 2 Leibniz-Institut für Werkstofforientierte Technologien (IWT), \\ Badgasteiner Straße 3, 28359 Bremen, Germany \\ 3 University of Bremen, MAPEX Center for Materials and Processes, \\ Postbox 330440, 28359 Bremen, Germany \\ c.vanselow@bimaq.de
}

\begin{abstract}
Summary:
An extension from qualitative to quantitative flow measurements of the cooling lubricant supply during grinding is achieved using shadowgraphy and particle image velocimetry. As a result, the measurement results in a grinding machine show the general feasibility of optical measurements despite severe optical disturbances in the two-phase flow. While using tracer particles enables closer measurements to the grinding wheel, both measurement approaches provide similar results. This enables investigations for the identification of flow characteristics, which are responsible for an efficient cooling.
\end{abstract}

Keywords: Optical flow field measurement, shadowgraphy, particle image velocimetry, grinding, liquid jet flow

\begin{abstract}
Motivation
In order to prevent grinding burn, coolant flows are used for lubrication and cooling of grinding processes [1], which are an important manufacturing step for metallic and optical components. However, only qualitative coolant flow characteristics were analyzed to optimize the jet inflow conditions yet [2]. A deeper insight in the cooling process can be obtained with quantitative flow field measurements enabling a recourse conservation, a more efficient process control and a better surface finish.
\end{abstract}

\section{State of the art}

Besides analyzing the jet exit velocity, the angle of impact on the grinding wheel and the nozzle shape [2], qualitative flow visualizations were conducted with shadowgraphy imaging in order to optimize the cooling efficiency in the grinding process [3]. Shadowgraphy uses a homogeneous background illumination to observe the fluid with a camera. Curvatures in the refractive index field of the fluid lead to deflected light rays that propagate from the background to the camera and corresponding parts of the camera image appear only weak or not at all illuminated. Shadowgraphy was used to determine the fluid breakup length of different nozzle flows. However, shadowgraphy is also capable of quantitative flow velocity field measurements as it is shown in [4] for a turbulent sonic helium jet in air and a 2d turbulent boundary layer at Mach 3. Here the visible turbulence structures in the flow, as they also occur in the visualized coolant flows in grinding processes, are tracked in successive images by an algorithm based on cross-correlation. In principle, this allows a measurement of two velocity components of the coolant flow field in the grinding process.

Particle image velocimetry (PIV) utilizes tracer particles which follow the flow with negligible slip [5]. The particles are illuminated with a light sheet from a double pulsed laser and observed by a camera. A cross-correlation algorithm is used to measure two components of the flow velocity field and may also be capable of measuring the coolant flow in the grinding process. However, in contrast to shadowgraphy, PIV measurements are disturbed by inhomogeneous refractive index fields which lead to an unpredictable measurement error [6].

\footnotetext{
Approach

Shadowgram imaging and PIV are used as complementary measurement techniques to measure the flow velocity fields of the coolant in the grinding machine for the first time. As a first step, the considered coolant flow is measured without a workpiece.

The signal evaluated with shadowgraphy is generated by undisturbed light rays propagating from the homogeneous background illumination through the flow field. In contrast, PIV evaluates the light reflected from particles located inside the light sheet, which can be disturbed on its
} 
way to the camera by the inhomogeneous refractive index field of the flow field. Thus, the PIV signal can be disturbed by light refraction whereby most of the signal for shadowgraphy is not affected by inhomogeneous refractive index fields. However, the image contrast vanishes in the shadowgram, if the illuminated flow field is too thick and the observed turbulence structures conceal each other. Here, PIV with a thin light sheet is advantageous since the light does not have to propagate through the whole flow field. As a result, when both techniques are used, shadowgraphy serves as a reference measurement when a sufficient contrast is available, and PIV allows velocity estimations when shadowgraphy is not working.

\section{Results}

The average velocity fields of different coolant volume flows are measured, whereby 1000 shadowgraphy images each are recorded by a high-speed camera with a repetition rate of $12 \mathrm{kHz}$. Furthermore, 700 PIV single measurements with a repetition rate of $15 \mathrm{~Hz}$ and a laser light pulse interval of 20 us are performed. The light sheet optics are integrated into the grinding machine with a waterproof box and the laser light is provided by a light guiding arm. The velocity fields are evaluated by a commercial iterative algorithm with an adaptive interrogation window size using a minimum of $32 \times 32 \mathrm{px}^{2}$. The measured velocity field with PIV for a volume flow of $55 \mathrm{Lmin}^{-1}$ and the difference $\Delta v$ to the measured velocity field with shadowgraphy imaging is depicted in Fig. 1. The free jet flows from $x=y=0 \mathrm{~cm}$ to the positive $x$-direction and impinges on the rotating grinding wheel at about $x=25 \mathrm{~cm}$, where the grinding wheel rotates with a circumferential velocity of $25 \mathrm{~ms}^{-1}$. The difference $\Delta v$ between the measurement techniques is in most elements of the measurement volume near zero, which indicates valid measurement results. However, at the region near the grinding wheel surface occur high differences in the measured velocity. Here, the contrast in the shadowgraphy images does not allow an evaluation of the fluid velocity. As a result, the velocity field can be measured closer to the rotating grinding wheel with PIV.

As an example, in Fig. 2a), a raw image is displayed with the evaluated velocity field. The transition between grinding wheel and fluid is hardly noticeable. Especially in the region at the grinding wheel surface with $x<80 \mathrm{~cm}$, a lack of velocity vectors is apparent. Here, the PIV raw image displayed in Fig. 2b) shows a much higher contrast. There are no seeding particles observables, but the velocity evaluation works using the droplets and the turbulence structures in the flow for the correlation. As a result, the velocity field of the coolant flow was measured for the first time. Thus, an experimental flow study to identify the flow characteristics responsible for an efficient cooling is feasible.

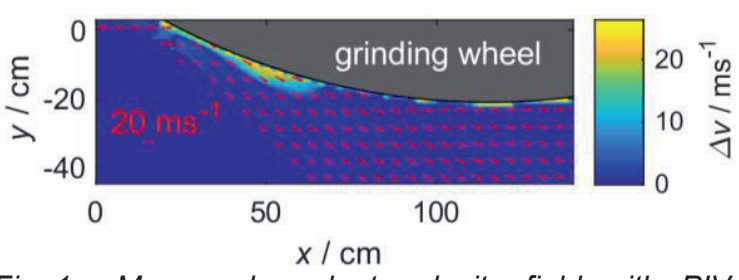

Fig. 1. Measured coolant velocity field with PIV visualized by the arrows, while the color indicates the difference $\Delta v$ to the measured velocity with shadowgraphy imaging.
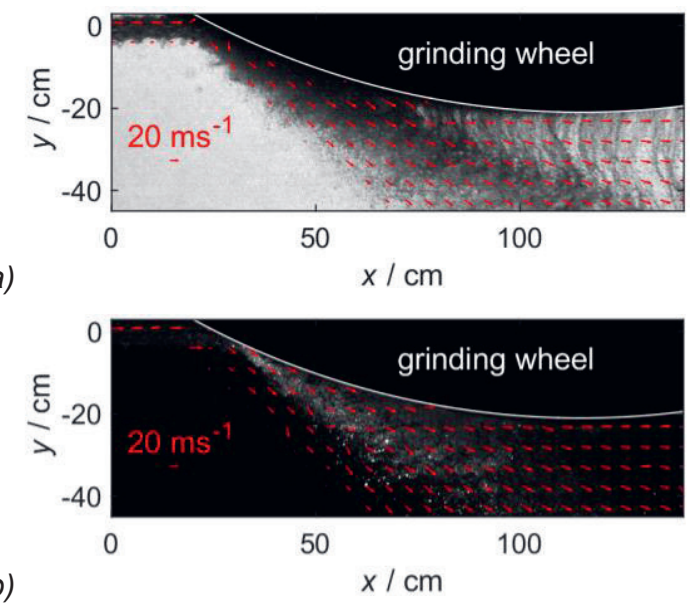

Fig. 2. a) Shadowgraphy and b) PIV raw images together with the resulting velocity flow field.

\section{Acknowledgement}

This research is funded by Deutsche Forschungsgemeinschaft (DFG, German Research Foundation) project number 415003387.

\section{References}

[1] E. Brinksmeier, D. Meyer, A. Huesmann-Cordes, C. Herrmann, CIRP Annals 64(2), 605-628 (2015); doi: 1016/j.cirp.2015.05.003

[2] C. Heinzel, D. Meyer, B. Kolkwitz, J. Eckebrecht, CIRP Annals 64(1), 333-336 (2015); doi: 10.1016/j.cirp.2015.04.009

[3] P. Geilert, C. Heinzel, A. Wagner, Inventions 2(4), 27 (2017); doi: 10.3390/inventions2040027

[4] D. R. Jonassen, G.S. Settles, M.D. Tronosky, Optics and Lasers in Engineering 44(3-4), 190-207 (2006); doi: 10.1016/j.optlaseng.2005.04.004

[5] M. Raffel, C. Willert, J. Kompenhans Particle Image Velocimetry: A Practical Guide (Experimental Fluid Mechanics), Springer, 2002; doi: 10.1007/978-3-319-68852-7

[6] C. Vanselow, A. Fischer, Optics and Lasers in Engineering 107, 221-230 (2018); doi: 0.1016/j.optlaseng.2018.03.020 